\title{
Role of Exosomal miRNAs in Heart Failure
}

\author{
Ruicong Xue ${ }^{1,2,3}$, Weiping Tan ${ }^{4}$, Yuzhong Wu ${ }^{1,2,3}$, Bin Dong 1,2,3, Zengshuo Xie ${ }^{1,2,3}$, \\ Peisen Huang ${ }^{1,2,3}$, Jiangui He ${ }^{1,2,3}$, Yugang Dong ${ }^{1,2,3 *}$ and Chen Liu ${ }^{1,2,3 *}$ \\ ${ }^{1}$ Department of Cardiology, First Affiliated Hospital of Sun Yat-sen University, Guangzhou, China, ${ }^{2}$ NHC Key Laboratory of \\ Assisted Circulation, Sun Yat-sen University, Guangzhou, China, ${ }^{3}$ National-Guangdong Joint Engineering Laboratory for \\ Diagnosis and Treatment of Vascular Diseases, First Affiliated Hospital of Sun Yat-sen University, Guangzhou, China, \\ ${ }^{4}$ Department of Respiratory, the First Affiliated Hospital of Sun Yat-sen University, Guangzhou, China
}

\section{OPEN ACCESS}

Edited by:

Christian Schulte,

University Heart and Vascular Center Hamburg (UHZ), Germany

Reviewed by:

Hamed Mirzaei,

Kashan University of Medical

Sciences, Iran

Peifeng Li,

Qingdao University, China

*Correspondence:

Yugang Dong

dongxg@mail.sysu.edu.cn

Chen Liu

liuch75@mail.sysu.edu.cn

Specialty section

This article was submitted to Cardiovascular Genetics and Systems

Medicine,

a section of the journal

Frontiers in Cardiovascular Medicine

Received: 10 August 2020 Accepted: 26 November 2020 Published: 17 December 2020

Citation:

Xue R, Tan W, Wu Y, Dong B, Xie Z, Huang P, He J, Dong Y and Liu C (2020) Role of Exosomal miRNAs in Heart Failure.

Front. Cardiovasc. Med. 7:592412. doi: 10.3389/fcvm.2020.592412
Heart failure is the terminal outcome of the majority of cardiovascular diseases, which lacks specific diagnostic biomarkers and therapeutic targets. It contributes to most of cardiovascular hospitalizations and death despite of the current therapy. Therefore, it is important to explore potential molecules improving the diagnosis and treatment of heart failure. MicroRNAs (miRNAs) are small non-coding RNAs that have been reported to be involved in regulating processes of heart failure. After the discovery of miRNAs in exosomes, the subcellular distribution analysis of miRNAs is raising researchers' attention. Growing evidence demonstrates that exosomal miRNAs may be promising diagnostic and therapeutic molecules for heart failure. This review summarizes the role of exosomal miRNAs in heart failure in the prospect of molecular and clinical researches.

Keywords: heart failure, exosomal miRNAs, cardiomyocyte hypertrophy, cardiac fibrosis, myocardial angiogenesis

\section{INTRODUCTION}

Heart failure is the terminal stage of various CVDs, with sustaining high morbidity and mortality contributing most to cardiac death (1). Despite of the current therapy strategy, the mortality and the re-hospitalization of heart failure are still high and no specific drugs are found to reverse heart failure once initiated. Therefore, exploring the pathogenesis of heart failure and labeling molecules closely related to the occurrence and development of heart failure are quite important, since these actions can post significant impact on early detection and treatment of the disease which can be prevented from adverse events.

MicroRNAs (miRNAs) are small non-coding RNAs that have been discovered for over two decades. MiRNAs mainly function as negative regulators of post-transcription of gene expressions through hybridization of $3^{\prime}$ untranslated regions ( $3^{\prime}$ UTRs) or open reading frames (ORFs) of the targeted mRNAs. Although they don't encode any protein, they are widely involved in the modulation of various genes at post-transcription level and thereby impacting various biological processes such as autophagy, oxidative stress and so on (2-5). Exosomes are bilayer membrane extracellular vesicles (EVs) carrying lipids, proteins and nucleic acids. They can be secreted by almost all eukaryotic cells through the fusion of multivesicular bodies and plasma membranes. They are released to extracellular matrix and responsible for carrying biological regulatory information [proteins, lipids, DNAs, and RNAs, especially miRNAs (6)] to the target cells in both surrounding and distant sites and organs, serving as paracrine signaling mediating intercellular communications. Exosomes transport intercellular information via four mechanisms: (1) Exosomal membrane proteins bind to the ligand-receptors in membranes of the target cells directly, activating certain signaling pathways; (2) Exosomal membrane proteins are splintered into pieces, releasing 
soluble ligands that bind to receptors of recipient cells; (3) Exosomes membranes directly fuse with the membranes of the target cells; (4) Internalization of exosomes by endocytic processes such as phagocytosis and macropinocytosis (7).

MiRNAs distribute widely in cellular components, and recent studies revealed that miRNAs also exist in exosomes (exosomal miRNAs, exo-miRNAs) (8). Furthermore, recent evidences revealed that miRNAs can be isolated easily and steadily from many kinds of tissues and body fluids including circulation, urine and saliva. In circulation, the detective miRNAs are mainly in the forms of exosomal miRNAs. The miRNAs are steady due to the protection of exosomes, whereas miRNAs in free forms will be degraded by nuclease (9). The discovery of exosomal miRNAs especially in circulation broadens functions of miRNAs to intercellular paracrine signaling mediators and disease biomarkers. In heart failure, mounting evidences have suggested that miRNAs participate in its pathogenesis and progression. However, attention focused on the component analysis of miRNAs and the related mechanisms in heart failure are still lacking. This review summarizes the recent evidences and progress of exosomal miRNAs in heart failure.

\section{EXOSOMAL MIRNAS: ORIGIN, FUNCTION AND MECHANISM IN CVDs}

Exosomal miRNAs are important biological products in exosomes, which are widely involved in information transmission between cells. It is worth noting that miRNAs do not enter exosomes randomly, but specific miRNAs are selectively entered into exosomes. Mature miRNA enters exosomes through four ways: (1) the miRISC related pathway; (2) sphyngomyelinase 2 dependent pathway; (3) heterogeneous nuclear ribonucleoproteins dependent pathway; (4) 3'miRNA sequence-dependent pathway. These are important ways for exosomal miRNAs to achieve directional information exchange between cells. Exosomal miRNAs participate in a variety of basic cell functions through paracrine or endocrine mechanisms, including cell proliferation, apoptosis, cytokine production, immune regulation and metastasis $(10,11)$. Under pathophysiological conditions, the content of specific exosomal miRNAs will also change (12). This makes evaluating levels of exosomal miRNAs a way for us to diagnose (13-17) and treat diseases (18).

Abbreviations: miRNAs, MicroRNAs; $3^{\prime}$ UTRs, $3^{\prime}$ untranslated regions; ORFs, Open reading frames; EVs, Extracellular vesicles; ECs, Endothelial cells; CVDs, Cardiovascular diseases; MI, Myocardial infarction; ECM, Extracellular matrix; MMP, Matrix Metalloproteinases; GSK3 $\beta$, Glycogen synthase kinase 3 $\beta$; mTOR, Mammalian target of rapamycin; Nrf2, Nuclear factor erythroid2-related factor 2; ARE, Antioxidant response elements; SOS-1, Son of Sevenless-1; Dyrk, Dualspecificity tyrosine-regulated kinases; CTGF, Connective Tissue Growth Factor; TA, Tibialis anterior; AHF, Acute heart failure; HFrEF, Heart failure with reduced ejection fraction; LVFS, Left ventricular fraction shortening; LVEF, Left ventricular ejection fraction; LAD, Left atrial diameter; LVDD, Left ventricular diastolic diameters; LVSD, Left ventricular systolic diameters; DCM, Dilated cardiomyopathy; ESCs, Embryonic stem cells; iPSCs, Induced pluripotent stem cells; CPCs, Cardiac progenitor cells; MSCs, Mesenchymal stem cells; BMSC, Bone marrow-derived MSC; hECT, Human engineered cardiac tissue; hMSCs, Human mesenchymal stem cells.
In the cardiovascular field, exosomal miRNAs normally play the role as cardiovascular cells communicators. Almost all kinds of cardiovascular cells are proved to release and receive exosomal miRNAs, indicating their potential regulatory roles in CVDs. Cardiomyocytes and fibroblasts, the most prevalent cell types in the heart, are the main source of exosomal miRNAs. Other than that, ECs and macrophages are also proved to release exosomal miRNAs in different CVDs (19). Depending on the source of exosomal miRNAs, target cells and the disease settings, these exosomal miRNAs act as different regulators on various molecular pathways in different CVDs [such as myocardial infarction, cardiomyopathy, arrhythmia, atherosclerosis and so on (20), Table 1], and the mechanisms involved are various. However, none of the studies successfully illustrate how exosomal miRNAs fulfill their specificity and further illustrated the way specific miRNA incorporate into exosomes. They use exosomes as non-selective transporters rather tissue/cell specific transmitters. Based on the above, we believe that there is still a long way for the research of exosomal miRNAs in CVDs.

\section{EXOSOMAL MIRNAs IN HEART FAILURE}

Heart failure is the terminal stage of almost all cardiovascular disorders mentioned above. It is characterized by a series of pathological processes including cardiomyocyte hypertrophy, cardiac fibrosis, impaired myocardial angiogenesis. Owing to the unique traits of exosomal miRNAs, investigating how they affect the pathogenesis of heart failure is meaningful.

\section{Exosomal miRNAs in Cardiomyocyte Hypertrophy as Regulators}

A series of miRNAs have been demonstrated to modulate the pathogenesis of cardiac hypertrophy which is the key mechanism of heart failure. MiR-199a targets the glycogen synthase kinase $3 \beta$ (GSK3 $\beta) /$ mammalian target of rapamycin (mTOR) complex signaling pathway to impair cardiomyocyte autophagy and thereby enhancing cardiac hypertrophy (miR199a impairs autophagy and induces cardiac hypertrophy through mTOR activation). In the model of heart failure, cardiacspecific overexpression of miR-221 significantly deteriorates cardiac function and promotes heart failure and mTOR axisrelated autophagy (29). However, few of the studies above have analyzed the component and the origin of the miRNAs involved in the regulation of cardiac hypertrophy. Furthermore, how miRNAs in non-cardiomyocytes exert their anti-hypertrophic effects on cardiomyocytes remains unexplained.

Recently, more and more evidences indicate that exosomal miRNAs might illustrate the issues above well Figure 1. Under pro-hypertrophic stresses, exosomal miRNAs from various kinds of cardiovascular cells target at cardiomyocytes, initiating or inhibiting cardiac hypertrophy. Based on the origin of exosomal miRNAs, there are basically three kinds of mechanisms involved: (1) Communication among cardiomyocytes: In MI model, exo-miRNA-133a level was significantly downregulated in cardiomyocytes in the infarct and peri-infarct areas. Exo-miR-133a released from ischemic cardiomyocytes 
TABLE 1 | Classification and mechanisms of exosomal miRNAs in CVDs according to the source of cell type.

\begin{tabular}{|c|c|c|c|c|c|c|}
\hline $\begin{array}{l}\text { Source of } \\
\text { exosomes }\end{array}$ & Type of CVD & Exo-miRNAs & Target cell & $\begin{array}{l}\text { Target molecular } \\
\text { pathway }\end{array}$ & Mechanisms & References \\
\hline \multirow[t]{2}{*}{ Cardiomyocytes } & $\begin{array}{l}\text { Chronic heart } \\
\text { failure }\end{array}$ & Exo-miR-217 & $\begin{array}{l}\text { Cardiac } \\
\text { fibroblast }\end{array}$ & $\begin{array}{l}\text { phosphatase and } \\
\text { tension homolog } \\
\text { deleted on } \\
\text { chromosome ten } \\
\text { (PTEN) }\end{array}$ & $\begin{array}{l}\text { Induce cardiac } \\
\text { fibrosis and } \\
\text { cardiac } \\
\text { dysfunction }\end{array}$ & (21) \\
\hline & $\begin{array}{l}\text { Diabetic } \\
\text { hearts }\end{array}$ & Exo-miR-320 & ECs & $\begin{array}{l}\text { Down-regulated its } \\
\text { target genes } \\
\text { (IGF-1, Hsp20, } \\
\text { and Ets2) }\end{array}$ & Anti-angiogenic & $(22)$ \\
\hline ECs & $\begin{array}{l}\text { Peripartum } \\
\text { cardiomyopathy }\end{array}$ & $\begin{array}{l}\text { Exo-miR- } \\
146 a\end{array}$ & Cardiomyocytes & $\begin{array}{l}\text { Downregulation of } \\
\text { Erbb4, Nras, } \\
\text { Notch1, and Irak1 }\end{array}$ & $\begin{array}{l}\text { Decrease } \\
\text { metabolic activity }\end{array}$ & (23) \\
\hline \multirow[t]{2}{*}{$\begin{array}{l}\text { Cardiac } \\
\text { fibroblasts }\end{array}$} & $\begin{array}{l}\text { Cardiac } \\
\text { hypertrophy }\end{array}$ & $\begin{array}{l}\text { Exo-miR-21- } \\
3 p\end{array}$ & Cardiomyocytes & $\begin{array}{l}\text { Silence sorbin and } \\
\text { SH3 } \\
\text { domain-containing } \\
\text { protein } 2 \\
\text { (SORBS2) and } \\
\text { PDZ and LIM } \\
\text { domain } 5 \\
\text { (PDLIM5) }\end{array}$ & $\begin{array}{l}\text { Induce cardiac } \\
\text { hypertrophy }\end{array}$ & (19) \\
\hline & $\begin{array}{l}\text { Atrial } \\
\text { fibrillation }\end{array}$ & $\begin{array}{l}\text { Exo-miR-21- } \\
3 p\end{array}$ & Cardiomyocytes & $\begin{array}{l}\text { Induce Cav1.2 } \\
\text { expression }\end{array}$ & $\begin{array}{l}\text { Increase } \\
\text { vulnerability under } \\
\text { atrial fibrillation }\end{array}$ & (24) \\
\hline Macrophages & $\begin{array}{l}\text { Uremic } \\
\text { cardiomyopathy }\end{array}$ & Exo-miR-155 & Cardiomyocytes & $\begin{array}{l}\text { Repress factors of } \\
\text { the O class } \\
\text { (FoxO3a) }\end{array}$ & $\begin{array}{l}\text { Induce cardiac } \\
\text { hypertrophy and } \\
\text { fibrosis }\end{array}$ & $(25)$ \\
\hline Fat cells & $\begin{array}{l}\text { Cardiac } \\
\text { hypertrophy }\end{array}$ & Exo-miR-200 & Cardiomyocytes & $\begin{array}{l}\text { Decreased TSC1 } \\
\text { and active mTOR }\end{array}$ & $\begin{array}{l}\text { Induce cardiac } \\
\text { hypertrophy }\end{array}$ & $(26)$ \\
\hline AMSCs & $\begin{array}{l}\text { Atrial } \\
\text { fibrillation }\end{array}$ & $\begin{array}{l}\text { Exo-miR- } \\
320 d\end{array}$ & Cardiomyocytes & $\begin{array}{l}\text { Decreased } \\
\text { STAT3-dependent }\end{array}$ & $\begin{array}{l}\text { Induce cardiac } \\
\text { hypertrophy }\end{array}$ & $(27)$ \\
\hline Unknown & Atherosclerosis & $\begin{array}{l}\text { MiR 30e and } \\
\text { miR 92a }\end{array}$ & Unknown & $\begin{array}{l}\text { Negative correlate } \\
\text { with the plasma } \\
\text { ABCA1 level }\end{array}$ & $\begin{array}{l}\text { Might decrease } \\
\text { coronary } \\
\text { atherosclerosis }\end{array}$ & (28) \\
\hline
\end{tabular}

may be captured by cardiomyocytes adjacent to the nonintact areas, exerting inhibitory effects on hypertrophy by reducing necrosis and apoptosis of cardiomyocytes (30). However, there are no other positive findings that exosomal miRNAs released from cardiomyocytes targeted at cardiomyocytes. We speculate that ligand-receptors for specific exosomal miRNAs from cardiomyocytes are lacking in other cardiomyocytes under the same pathological condition. (2) Communication between cardiac fibroblasts and cardiomyocytes: Cardiac fibroblasts account for $\sim 60-70 \%$ of cardiac cells, which makes them sensitive transductors mediating the pro-hypertrophic signals via exosomal-miRNAs. Evidence that miRNA-enriched exosomes are involved in dysregulation of Nrf2-ARE by mediating intercellular communication between fibroblasts and cardiomyocytes supports the communication between cardiac fibroblasts and cardiomyocytes via exosomal miRNAs (31). Clues that murine cardiomyocyte hypertrophy could be induced by coculturing with cardiac fibroblasts or conditioned fibroblast media further indicates communication between cardiac fibroblasts and cardiomyocyte in cardiac hypertrophy $(32,33)$. Cardiac fibroblasts secrete exosomes enriched with miR-21-3p, inducing cardiac hypertrophy by targeting recipient cardiomyocytes (19). MiR-21, a proven regulator of fibroblasts biology, is increased in failing hearts, implicating its potential role in heart failure. However, overexpressing or inhibiting miR-21 in cardiomyocytes doesn't influence the hypertrophic phenotype, raising the question that the origin or distribution of miR-21 might be involved. Further evidences suggest that miR-21 expressions are extremely high in exosomes and miR-21 expressions are higher in donor fibroblasts, indicating that miR-21 is packed into exosomes. Furthermore, exo-miR-21 derived from fibroblast is proved to be absorbed into cardiomyocytes and subsequently decreases SORBS2 and PDLIM5 in cardiomyocytes, ultimately inducing cardiac hypertrophy. Thus, exo-miR-21, rather than miR-21, is a key mediator transferring pro-hypertrophy information from cardiac fibroblasts to cardiomyocytes (19). MiR-217 was also proved to be a potent marker of chronic heart failure, evidenced by increased expressions in heart failure and its overexpression pressure overload-induced cardiac hypertrophy. Further component analysis proved that cardiomyocyte-derived exosomes containing miR-217 enhanced proliferation of fibroblasts in vitro, suggesting that exosomal miR-217 was 


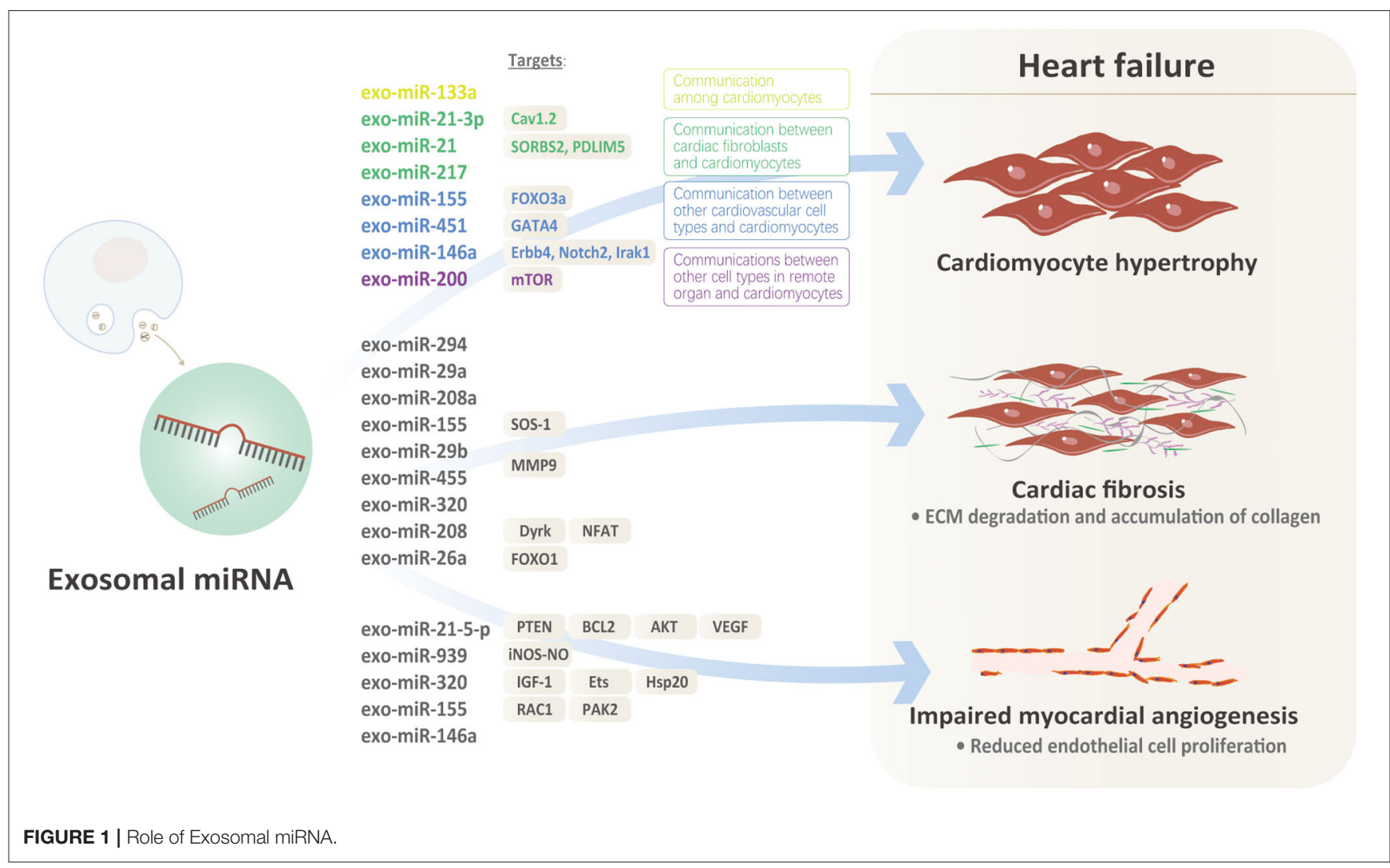

not only a potent marker but a promising therapeutic target for chronic heart failure (21). (3) Communications between other cardiovascular cell types and cardiomyocytes: Exosomes enriched with miR-155 which were derived from macrophages promoted cardiac hypertrophy and fibrosis by activating the prohypertrophic pathway FOXO3a in uremic mice (25). In acute MI, exosomal miRNAs also acted in cell-cell communication between cardiomyocytes and local or remote stem cells. For example, exosomes enriched with miR-451 that derived from cardiac progenitor cells protected cardiomyocytes from oxidative stress by targeting GATA4, thereby maintaining cardiac function. ExomiR-146a extracted from ECs were absorbed by cardiomyocytes and subsequently targeted on Erbb4, Notch2, Irak1, mediating the progression of heart failure through metabolic pathways (23). (4) Communications between other cell types in remote organs and cardiomyocytes: A recent research proved that PPAR- $\gamma$ activation in adipocytes from adipose tissues increased the secretion of miRNA-200-contained exosomes which promoted cardiomyocyte hypertrophy through the mTOR pathway (26).

Exosomal miRNAs are involved in the regulation of cardiac hypertrophy due to various etiology. However, based on the current evidences, the defined role of exosomal miRNAs in cardiac hypertrophy differs across disease settings and the sources, not to mention the miscellaneous mechanisms involved. We still lack further research illustrating how exosomes ensure the specificity for certain miRNAs targeting at specific cell types.

\section{Exosomal miRNAs in Cardiac Fibrosis as Regulators}

Cardiac fibrosis, characterized by ECM degradation and accumulation of collagen due to proliferation of cardiac fibroblasts, is another important mechanism of heart failure. MiRNAs have been reported to regulate cardiac fibrosis. For example, miR-19a-3p and miR-19b-3p targeted TGF- $\beta$ to inhibit epithelial-mesenchymal transition, ECM production and the invasion of human cardiac fibroblasts, thereby exhibiting the anti-fibroblast effect (34). More lights are shed on the regulation of cardiac fibrosis by exosomal miRNAs. Exosomal miR-294 from embryonic stem cells were proved to inhibit fibrosis after MI, thereby preventing MI-induced heart failure. Elevated exo-miR-29a in marginal ischemic area transferred the intercellular information among cardiomyocytes, mediating the antifibrotic effect and preventing ventricular dysfunction and heart failure (35). MiR-208a was upregulated in cardiomyocytes and cardiomyocyte-derived exosomes in cardiac fibrosis (36).

Mechanically, exosomal miRNAs from diverse sources participate in the regulation of different parts of cardiac fibrosis Figure 1. Macrophage-derived exo-miR-155 inhibited the proliferation of cardiac fibroblasts after MI by inhibiting the SOS-1, a critical modulator of RAS activation in mice (37). Cardiomyocyte-derived exosomes containing miR208a contributed to increased fibroblast proliferation and differentiation into myofibroblasts and thereby accelerating the progression of cardiac fibrosis (36). In type 2 diabetes 
mice, exercise significantly increased exosomes derived from cardiomyocytes which contain abundant miR-29b and miR455, thereby decreasing MMP9 and ECM degradation and resulting in the amelioration of cardiac fibrosis (38). In diabetic cardiomyopathy, exosomes derived from diabetic cardiomyocytes negatively affected proliferation and migration of ECs by transferring miRNA-320 and subsequently inhibited heart failure. This process was reversed by adding GW-4869, an exosome inhibitor (22). Exosomal miR-208 derived from cardiomyocytes inhibited the expression of Dyrk and phosphorylated NFAT to promote its nuclear export. Nuclear NFAT triggered fibrosis by inducing the expression of CTGF, Colla1, Col3a1, and a-SMA in cardiac fibroblasts (36). The evidences above have proved the fact that miRNAs in exosomes are important regulators of cardiac fibrosis and heart failure due to different etiologies. Injection of exo-miR-26a into tibialis anterior (TA) muscle significantly increased the expression of miR-26a in the heart and subsequently ameliorated cardiac fibrosis lesions. Furthermore, exo-miR-26a significantly blunted FOXO1 activation and inhibited cardiac fibrosis in the heart of mice with chronic kidney disease (39). This study demonstrated the strength of miRNAs encapsulated by exosomes for its tissue specificity, however, how exosomes guarantee this specificity needs illustrating. Furthermore, whether this specificity was due to the encapsulation of exosomes or miRNAs themselves are yet unknown.

\section{Exosomal miRNAs in Impaired Myocardial Angiogenesis as Regulators}

Besides of the pathologic changes in heart tissue, aberrant angiogenesis contributes to the acceleration from compensated cardiac hypertrophy to heart failure due to decreasing capillary angiogenesis and cardiomyocyte energy supply (40). ECs proliferation is the key factor in this process (41). Exosomal miRNAs have been proved to regulate angiogenesis in other diseases Figure 1, but how they affect angiogenesis in heart failure is not fully and directly illustrated.

Exo-miR-21-5-p from healthy hearts inhibited PTEN and BCL2 in cardiomyocytes and ECs and activated the AKT and VEGF pathways to promote cardiomyocyte proliferation and promote angiogenesis (42). Exosomes containing miR-939 from hearts in patients with myocardial ischemia significantly promoted angiogenesis through iNOS-NO pathway (43). However, the source of exosomal miRNAs in the researches above might need further study. In the subsequent studies, they perfectly demonstrated the sources, target cells and the mechanisms of exosomal miRNAs in the regulation of angiogenesis. Wang et al. discovered that exosomes isolated from diabetic cardiomyocytes significantly reduced angiogenesis in the diabetic myocardium via the exosomal transfer of miR-320 into ECs and subsequent decrease of IGF-1, Ets and Hsp20 (22). In MI, M1-like macrophages released a large number of proinflammatory exosomes, transferring miR-155 to ECs to inhibit angiogenesis by down-regulating Rac family small GTPase 1 and p21 activates kinase 2 (44). Interestingly, we discovered the same miRNA might exert completely opposite regulatory effects in angiogenesis in the process of heart failure. MiR-146a in the exosomes derived from ECs significantly decreased ECs proliferation and inhibited microvascular regeneration and impaired systolic/diastolic function in cardiomyopathy (23). However, exosomal miR-146a derived from cardiosphere cells promoted myocardial angiogenesis and significantly improved heart failure after acute MI (45). Some scholars believe that these contradictions may be related to different periods of heart failure. In the early stage, cardiac exosomes transmitted cardioprotective miRNAs, and in the terminal stages, exosomal miRNAs may play a damaging role (46). In addition, we also assume that the seemingly opposite results might be attributed to the origin of miRNA and different etiologies of heart failure. Further mechanistic research demonstrating this contradiction is required.

\section{APPLICATION OF EXOSOMAL MIRNAS IN HEART FAILURE: BIOMARKERS AND THERAPEUTIC TARGETS}

The exploration of new biomarkers of heart failure is critical for the diagnosis and treatment of heart failure. BNP and NT-proBNP are widely used in the clinical diagnosis of heart failure. Some newly found biomarkers such as ST2 and NGAL also become to be used in clinical. However, in the case of combination with some other pathological conditions, such as pulmonary embolism and renal dysfunction, the specificity of the biomarkers mentioned above could be compromised.

A variety of miRNAs in circulation are involved in pathogenesis or protection of heart failure in a dynamic and stage-specific way and therefore they are potential biomarkers of heart failure. Circulating miR-23a, miR-23b, miR-24, miR195 , and miR-214 were found increased and circulating miR423-5p, miR-320, and miR-22 were found decreased in chronic heart failure $(47,48)$. As for acute heart failure (AHF), miR423-5p was proved to differentially express between heart failure patients and controls, which might serve as a biomarker distinguishing AHF and other causes of dyspnea (49). MiR210 in plasma was also proved to be elevated in heart failure patients with NYHA III-IV compared with those with NYHA II and controls (50). Other differential expression circulating miRNAs in AHF includes down-regulated miR-103, miR-1423p, miR-30b, miR-342-3p and up-regulated miR-499 (51, 52). Furthermore, miRNAs were also proved to have potential ability to evaluate prognosis. Decreased levels of miR-18a-5p and miR652-3p during hospitalization in heart failure patients were associated with a high predicted mortality within 180 days (53). Up-regulation of miR-192 was proved to be a prognostic marker predicting survival in ischemic heart failure with p53-mediated apoptosis involved (54).

However, none of the studies above analyzed the circulating components, especially exosome fraction and non-exosome supernatant. Wang et al. discovered that miR-425 and miR744 expressions were altered in plasma exosome samples in heart failure patients. A further functional study proved that they negatively regulated cardiac fibrosis by suppressing TGF $\beta 1$ 
expression, revealing their potential role in regulating and predicting heart failure (55). However, direct evidence of the regulatory role of miRNAs via exosomes was lacking in this study. Circulating miRNAs exist in the form of free miRNAs and exosome-encapsulated miRNAs. Free miRNAs in circulation are the byproduct of dead cells and their levels might be altered depending on the rates of tissue repair and age. On the contrary, exosomal miRNAs, making up $10-15 \%$ of circulating miRNAs, are more sensitive in providing stable and accurate patterns for disease diagnosis. However, the difficulty to isolate and detect circulating exosomal miRNAs limited their further application as heart failure biomarkers. As the technology develops, Turchinovich et al. firstly separated non-exosome supernatant fraction from exosome and Beg et al. constructed protocols to measure both free miRNAs and exosomal miRNAs in circulation, making it possible to proceed the biomarkers exploration focusing on exosomal miRNAs $(56,57)$. In the study of Beg's, they found an increase of miR-146a and miR486 (both had the poverty of anti-inflammation) in exosomes rather than those in plasma in the patients with heart failure compared to controls, indicating that exosomal miRNAs worth being further studied as heart failure biomarkers. Inspiringly, two studies supported the diagnostic value of exosomal miRNAs. Serum exo-miR-92b-5p was proved to be increased in HFrEF patients compared with controls, with a sensitivity of $71.4 \%$ and specificity of $83.3 \%$. Moreover, exo-miR-92b-5p was inversely correlated with the LVFS and LVEF, while positively correlated with LAD, LVDD and LVSD. Serum exo-miR-92b-5p is a potential diagnostic biomarker in AHF due to DCM, the elevation of which indicated the progression of AHF (58). In another study focused on ischemic HF post AMI discovered that both serum levels and exosomal levels of miR-192, miR194, and miR-34a were up-regulated in HF patients, confirming their diagnostic role in ischemic HF (59). However, this study did not compare the disparity between serum miRNAs and exosomal miRNAs regarding to their diagnostic value. In myxomatous mitral valve disease model of dogs, decreased exosomal miR-599 levels and increased exosomal miR-181c and miR-495 were found in chronic heart failure compared to controls, whereas no miRNA in total plasma remained statistically significant alteration if the false discovery rate was set $<15 \%$, indicating that exosomal miRNAs might be a more specific marker than total plasma miRNAs (60). The concern of the comparation of the diagnostic value between exosomal miRNAs and free miRNAs are raised consequently. Some studies supported that exosomal miRNAs exhibit more diagnostic and prognostic value than those in free form. For example, in stable coronary artery heart disease, miR-126 and miR-199a in exosomes were proved to be related with cardiovascular events rather than free miR-126 and miR-199a in plasma (61). However, a study opposed this opinion with the evidence that no differential expression of exosomal miRNA and freemiRNA in circulation were detected between chronic heart failure patients and controls (48). In a 30 patients' clinical study, the disparity of miRNA levels (miR-423-5p/320a/22/92b/17/532$3 \mathrm{p} / 92 \mathrm{a} / 30 \mathrm{a} / 21 / 101$ ) between patients with heart failure and controls were similar in exosomal fraction and unfractionated serum, suggesting that these miRNAs in exosome might not be ideal markers for heart failure. However, we doubt that the sample capacity in this study was small and the coverage of miRNA detected was limited (48). Further studies are desperately needed to demonstrate the diagnostic value of exosomal miRNAs in heart failure by expanding the number of patients enrolled and the miRNAs. Furthermore, the source of most of the exosomal miRNAs detected in circulation has not been further illustrated, limiting the mechanism exploration of the specific exosomal miRNAs.

\section{STEM CELLS-DERIVED EXOSOMAL miRNAs IN HEART FAILURE: OPPORTUNITIES AND CHALLENGES}

The stem cell-based therapies are becoming potential therapeutic strategies in many diseases and their influences radiate to heart failure, whereas the low efficiency of engraftment and potential tumorigenic risk of stem cell transplantation limits their prevalence. However, the emerging value of stem cellderived exosomes which might improve the efficiency of stem cell transplantation motivates the further exploration of stem cellbased therapies in heart failure. Exosomes derived from different kinds of stem cells, such as ESCs, iPSCs CPCs and MSCs, have been proved to exert no less protective properties but safer in operation than stem cell transplantation (62). As one of the major components in exosomes, several miRNAs in exosomes derived from stem cells have been identified to play important roles in heart failure. Intramyocardial delivery of exosomes enriched with miR-290-295 and miR-294 derived from ESCs significantly ameliorated fibrosis in acute MI. Exosomes from murine cardiac fibroblast-derived iPSCs, which were enriched with miR-21 and miR-210, have been proved to protect against oxidative stress and cardiac remodeling after acute MI in periinfarct regions. And the protective properties of miR-21 enriched exosomes were similar with miR-21 $(63,64)$. CPCs treatments help to improve cardiac repair and protect against damage and exosomes in CPCs are involved. Proangiogenic miR-17 and miR210 were up-regulated in CPC-derived exosomes under oxidative stress and thereby inhibited TGF- $\beta$ induced fibrosis, providing another profound evidence that miRNAs in exosomes might be a pivotal component in the stem cell therapy of heart failure (65). Researchers found that bone marrow-derived MSC (BMSC) from mice after ischemic preconditioning could secrete miR22 highly enriched exosomes, and in the MI mouse model, miR-22 targeted methyl CpG binding protein 2 (Mecp2) to reduce infarct size and cardiac fibrosis (66). After treating human engineered cardiac tissue (hECT) with exosomes derived from human mesenchymal stem cells (hMSCs), the level of miR-21-5p is significantly increased in hECT, while knockout of miR-21$5 \mathrm{p}$ in hMSCs reduced expression of pro-contraction and related calcium-treated genes in the hECT (67). These findings indicate that stem cell-derived exosomes contain a variety of miRNAs which play an important role in heart failure (Table 2). The use of these exosomes may contribute to the development of novel treatment strategies for heart failure in the future. 
TABLE 2 | The role of miRNAs in stem cell-derived exosomes in heart failure.

\begin{tabular}{|c|c|c|c|c|}
\hline MiRNA & Sources & Animal model & Function & References \\
\hline miR-290-295 & ESC & AMI & Ameliorated fibrosis in acute $\mathrm{MI}$ & (68) \\
\hline miR-294 & ESC & AMI & Ameliorated fibrosis in acute Ml & (68) \\
\hline miR-21 & iPSC & $\mathrm{Ml} / \mathrm{R}$ & $\begin{array}{l}\text { Protect against oxidative stress and } \\
\text { cardiac remodeling after acute MI/R }\end{array}$ & (64) \\
\hline miR-210 & iPSC & $\mathrm{Ml} / \mathrm{R}$ & $\begin{array}{l}\text { Protect against oxidative stress and } \\
\text { cardiac remodeling after acute } \mathrm{MI} / \mathrm{R}\end{array}$ & (64) \\
\hline miR-210 & $\mathrm{CPC}$ & $\mathrm{Ml} / \mathrm{R}$ & Inhibited fibrosis & (65) \\
\hline miR-22 & BMSC & $\mathrm{Ml} / \mathrm{R}$ & Reduce infarct size and cardiac fibrosis & (66) \\
\hline miR-21-5p & hMSC & hECT & $\begin{array}{l}\text { Promotes contraction and calcium } \\
\text { processing }\end{array}$ & (67) \\
\hline
\end{tabular}

\section{PROSPECT}

The types and levels of miRNAs in exosomes in the circulatory system are more closely related to heart failure compared to free miRNAs. Other than free miRNAs, their level changes may be related to the severity of heart failure. So, they can provide a more sensitive and specific diagnostic method for heart failure. However, there is evidence that no differential expression of exosomal miRNAs and free miRNAs in the circulation was detected between patients with chronic heart failure and the controls. Therefore, whether circulating exosomes miRNAs can be used as biomarkers for the diagnosis and prognosis of heart failure is still questionable. We need studies with larger number of patients and the kinds of miRNAs to determine: whether there are significant changes in the levels of circulating exosomal miRNA after heart failure, whether there is a correlation between these changes and the pathophysiological changes in heart failure, whether the sensitivity and specificity of these new diagnostic methods are better than those currently used in clinical practice.

Heart failure may affect circulating exosomal miRNAs, and exosomes derived from stem cells can also play an important role in heart failure. They may serve as a new strategy for treating or preventing heart failure in the future. However, this new strategy is in its infancy. There are still several hurdles before achieving clinical application (62): First of all, the purification and separation technology of exosomes is not

\section{REFERENCES}

1. Berezin A. Epigenetics in heart failure phenotypes. BBA Clin. (2016) 6:317. doi: 10.1016/j.bbacli.2016.05.005

2. Huang Z, Wu S, Kong F, Cai X, Ye B, Shan P, et al. MicroRNA-21 protects against cardiac hypoxia/reoxygenation injury by inhibiting excessive autophagy in H9c2 cells via the Akt/mTOR pathway. J Cell Mol Med. (2017) 21:467-74. doi: $10.1111 / \mathrm{jcmm} .12990$

3. Xu C, Hu Y, Hou L, Ju J, Li X, Du N, et al. $\beta$-Blocker carvedilol protects cardiomyocytes against oxidative stress-induced apoptosis by up-regulating miR-133 expression. J Mol Cell Cardiol. (2014) 75:11121. doi: 10.1016/j.yjmcc.2014.07.009

4. Li J, Donath S, Li Y, Qin D, Prabhakar BS, Li P. miR30 regulates mitochondrial fission through targeting p53 and perfect, it takes a long time and the efficiency is low (69). Secondly, exosomes contain a large number of biologically active factors, so they may cause adverse or side effects on cardiac tissue. Thirdly, due to the heterogeneous components in exosomes, it may show a potential risk for tumor formation and a risk of adverse immune response based on donor cell characteristics. Finally, due to the complex structure of exosomes, the therapeutic effect that exosomes produced during the disease is unknown. How exosomal miRNAs fulfill its specificity is yet fully understood. Therefore, the future clinical translation of exosomes for heart failure treatment still faces major challenges. We need to clearly analyze the various components in stemderived exosomes, clarifying their physiological effects on cardiac tissue, as well as their therapeutic and side effects on human body.

\section{AUTHOR CONTRIBUTIONS}

$\mathrm{RX}, \mathrm{ZX}, \mathrm{PH}$, and WT wrote the manuscript. YW drew the figure. JH, CL, and YD edited the manuscript. All authors contributed to the article and approved the submitted version.

\section{FUNDING}

The present study was funded by the National Natural Science Foundation of China (Nos. 81700344). the dynamin-related protein-1 pathway. PLoS Genet. (2010) 6:e1000795. doi: 10.1371/annotation/4050116d-8daa-4b5a-99e9-34cdd13f6a26

5. Khani P, Nasri F, Khani Chamani F, Saeidi F, Sadri Nahand J, Tabibkhooei A, et al. Genetic and epigenetic contribution to astrocytic gliomas pathogenesis. J Neurochem. (2019) 148:188-203. doi: 10.1111/jnc. 14616

6. Meng Y, Sun J, Wang X, Hu T, Ma Y, Kong C, et al. Exosomes: a promising avenue for the diagnosis of breast cancer. Technol Cancer Res Treat. (2019) 18:1077089069. doi: $10.1177 / 1533033818821421$

7. Turturici G, Tinnirello R, Sconzo G, Geraci F. Extracellular membrane vesicles as a mechanism of cell-to-cell communication: advantages and disadvantages. Am J Physiol. (2014) 306:C621-33. doi: 10.1152/ajpcell.00228.2013

8. Aghabozorgi AS, Ahangari N, Eftekhaari TE, Torbati PN, Bahiraee A, Ebrahimi R, et al. Circulating exosomal miRNAs in cardiovascular disease 
pathogenesis: new emerging hopes. J Cell Physiol. (2019) 234:21796809. doi: $10.1002 / j c p .28942$

9. Zampetaki A, Willeit P, Drozdov I, Kiechl S, Mayr M. Profiling of circulating microRNAs: from single biomarkers to re-wired networks. Cardiovasc Res. (2012) 93:555-62. doi: $10.1093 / \mathrm{cvr} / \mathrm{cvr} 266$

10. Sadri Nahand J, Moghoofei M, Salmaninejad A, Bahmanpour Z, Karimzadeh $M$, Nasiri $M$, et al. Pathogenic role of exosomes and microRNAs in HPV-mediated inflammation and cervical cancer: a review. Int $J$ Cancer. (2020) 146:305-20. doi: 10.1002/ijc.32688

11. Huang-Doran I, Zhang C, Vidal-Puig A. Extracellular vesicles: novel mediators of cell communication in metabolic disease. Trends Endocrinol Metab. (2017) 28:3-18. doi: 10.1016/j.tem.2016.10.003

12. Zhang J, Li S, Li L, Li M, Guo C, Yao J, et al. Exosome and exosomal microRNA: trafficking, sorting, and function. Genom Proteom Bioinform. (2015) 13:17-24. doi: 10.1016/j.gpb.2015. 02.001

13. Nahand JS, Mahjoubin-Tehran M, Moghoofei M, Pourhanifeh MH, Mirzaei HR, Asemi Z, et al. Exosomal miRNAs: novel players in viral infection. Epigenomics. (2020) 12:353-70. doi: 10.2217/epi-2019-0192

14. Ghaemmaghami AB, Mahjoubin-Tehran M, Movahedpour A, Morshedi K, Sheida A, Taghavi SP, et al. Role of exosomes in malignant glioma: microRNAs and proteins in pathogenesis and diagnosis. Cell Comm Signal. (2020) 18:120. doi: 10.1186/s12964-020-00623-9

15. Aghdam AM, Amiri A, Salarinia R, Masoudifar A, Ghasemi F, Mirzaei H. MicroRNAs as diagnostic, prognostic, and therapeutic biomarkers in prostate cancer. Crit Rev Eukar Gene. (2019) 29:127-39. doi: 10.1615/CritRevEukaryotGeneExpr.2019025273

16. Pourhanifeh MH, Mahjoubin-Tehran M, Karimzadeh MR, Mirzaei HR, Razavi ZS, Sahebkar A, et al. Autophagy in cancers including brain tumors: role of MicroRNAs. Cell Comm Signal. (2020) 18:88. doi: 10.1186/s12964-020-00587-w

17. Hashemian SM, Pourhanifeh MH, Fadaei S, Velayati AA, Mirzaei H, Hamblin MR. Non-coding RNAs and exosomes: their role in the pathogenesis of sepsis. Mol Therapy Nucleic Acids. (2020) 21:51-74. doi: 10.1016/j.omtn.2020.05.012

18. Rezaei S, Mahjoubin-Tehran M, Aghaee-Bakhtiari SH, Jalili A, Movahedpour A, Khan $\mathrm{H}$, et al. Autophagy-related MicroRNAs in chronic lung diseases and lung cancer. Crit Rev Oncol Hematol. (2020) 153:103063. doi: 10.1016/j.critrevonc.2020.103063

19. Bang C, Batkai S, Dangwal S, Gupta SK, Foinquinos A, Holzmann A, et al. Cardiac fibroblast-derived microRNA passenger strand-enriched exosomes mediate cardiomyocyte hypertrophy. J Clin Investig. (2014) 124:213646. doi: 10.1172/JCI70577

20. Colpaert RMW, Calore M. MicroRNAs in cardiac diseases. Cells Basel. (2019) 8:737. doi: $10.3390 /$ cells 8070737

21. Nie X, Fan J, Li H, Yin Z, Zhao Y, Dai B, et al. miR-217 promotes cardiac hypertrophy and dysfunction by targeting PTEN. Mol Therapy Nucleic Acids. (2018) 12:254-66. doi: 10.1016/j.omtn.2018.05.013

22. Wang X, Huang W, Liu G, Cai W, Millard RW, Wang Y, et al. Cardiomyocytes mediate anti-angiogenesis in type 2 diabetic rats through the exosomal transfer of miR-320 into endothelial cells. J Mol Cell Cardiol. (2014) 74:13950. doi: 10.1016/j.yjmcc.2014.05.001

23. Halkein J, Tabruyn SP, Ricke-Hoch M, Haghikia A, Nguyen N, Scherr M, et al. MicroRNA-146a is a therapeutic target and biomarker for peripartum cardiomyopathy. J Clin Investig. (2013) 123:2143-54. doi: 10.1172/JCI64365

24. Li S, Gao Y, Liu Y, Li J, Yang X, Hu R, et al. Myofibroblast-derived exosomes contribute to development of a susceptible substrate for atrial fibrillation. Cardiology. (2020) 145:324-32. doi: 10.1159/000505641

25. Wang B, Wang Z, Ji J, Gan W, Zhang A, Shi H, et al. Macrophagederived exosomal Mir-155 regulating cardiomyocyte pyroptosis and hypertrophy in uremic cardiomyopathy. JACC. (2020) 5:148-66. doi: 10.1016/j.jacbts.2019.10.011

26. Fang X, Stroud MJ, Ouyang K, Fang L, Zhang J, Dalton ND, et al. Adipocytespecific loss of PPAR $\gamma$ attenuates cardiac hypertrophy. JCI Insight. (2016) 1:e89908. doi: 10.1172/jci.insight.89908

27. Liu L, Zhang H, Mao H, Li X, Hu Y. Exosomal miR-320d derived from adipose tissue-derived MSCs inhibits apoptosis in cardiomyocytes with atrial fibrillation (AF). Artif Cells Nanomed Biotechnol. (2019) 47:397684. doi: $10.1080 / 21691401.2019 .1671432$
28. Wang Z, Zhang J, Zhang S, Yan S, Wang Z, Wang C, et al. MiR-30e and miR92a are related to atherosclerosis by targeting ABCA1. Mol Med Rep. (2019) 19:3298-304. doi: 10.3892/mmr.2019.9983

29. Su M, Wang J, Wang C, Wang X, Dong W, Qiu W, et al. MicroRNA-221 inhibits autophagy and promotes heart failure by modulating the p27/CDK2/mTOR axis. Cell Death Differ. (2015) 22:986-99. doi: 10.1038/cdd.2014.187

30. Kuwabara Y, Ono K, Horie T, Nishi H, Nagao K, Kinoshita M, et al. Increased microRNA-1 and microRNA-133a levels in serum of patients with cardiovascular disease indicate myocardial damage. Circ Cardiovasc Genet. (2011) 4:446-54. doi: 10.1161/CIRCGENETICS.110.958975

31. Tian C, Gao L, Zimmerman MC, Zucker IH. Myocardial infarctioninduced microRNA-enriched exosomes contribute to cardiac Nrf2 dysregulation in chronic heart failure. Am J Physiol. (2018) 314:H928-39. doi: 10.1152/ajpheart.00602.2017

32. LaFramboise WA, Scalise D, Stoodley P, Graner SR, Guthrie RD, Magovern JA, et al. Cardiac fibroblasts influence cardiomyocyte phenotype in vitro. Am J Physiol. (2007) 292:C1799-808. doi: 10.1152/ajpcell.00166.2006

33. Fredj S, Bescond J, Louault C, Potreau D. Interactions between cardiac cells enhance cardiomyocyte hypertrophy and increase. J Cell Physiol. (2005) 202:891-9. doi: 10.1002/jcp.20197

34. Zou M, Wang F, Gao R, Wu J, Ou Y, Chen X, et al. Autophagy inhibition of hsa-miR-19a-3p/19b-3p by targeting TGF- $\beta$ R II during TGF$\beta 1$-induced fibrogenesis in human cardiac fibroblasts. Sci Rep. (2016) 6:24747. doi: $10.1038 /$ srep 24747

35. Yamaguchi $T$, Izumi $Y$, Nakamura $Y$, Yamazaki $T$, Shiota $M$, Sano $S$, et al. Repeated remote ischemic conditioning attenuates left ventricular remodeling via exosome-mediated intercellular communication on chronic heart failure after myocardial infarction. Int J Cardiol. (2015) 178:2396. doi: 10.1016/j.ijcard.2014.10.144

36. Yang J, Yu X, Xue F, Li Y, Liu W, Zhang S. Exosomes derived from cardiomyocytes promote cardiac fibrosis via myocyte-fibroblast cross-talk. Am J Transl Res. (2018) 10:4350-66.

37. Wang C, Zhang C, Liu L, AX, Chen B, Li Y, et al. Macrophage-derived mir-155-containing exosomes suppress fibroblast proliferation and promote fibroblast inflammation during cardiac injury. Mol Therapy. (2017) 25:192204. doi: 10.1016/j.ymthe.2016.09.001

38. Chaturvedi P, Kalani A, Medina I, Familtseva A, Tyagi SC. Cardiosome mediated regulation of MMP9 in diabetic heart: role of mir29b and mir455 in exercise. J Cell Mol Med. (2015) 19:2153-61. doi: 10.1111/jcmm. 12589

39. Wang B, Zhang A, Wang H, Klein JD, Tan L, Wang Z, et al. miR26a limits muscle wasting and cardiac fibrosis through exosome-mediated microRNA transfer in chronic kidney disease. Theranostics. (2019) 9:186477. doi: 10.7150/thno.29579

40. Gogiraju R, Bochenek ML, Schäfer K. Angiogenic endothelial cell signaling in cardiac hypertrophy and heart failure. Front Cardiovasc Med. (2019) 6:20. doi: $10.3389 /$ fcvm.2019.00020

41. Zhabyeyev P, Gandhi M, Mori J, Basu R, Kassiri Z, Clanachan A, et al. Pressure-overload-induced heart failure induces a selective reduction in glucose oxidation at physiological afterload. Cardiovasc Res. (2013) 97:67685. doi: $10.1093 / \mathrm{cvr} / \mathrm{cvs} 424$

42. Qiao L, Hu S, Liu S, Zhang H, Ma H, Huang K, et al. microRNA21-5p dysregulation in exosomes derived from heart failure patients impairs regenerative potential. J Clin Investig. (2019) 129:2237-50. doi: 10.1172/JCI123135

43. Li H, Liao Y, Gao L, Zhuang T, Huang Z, Zhu H, et al. Coronary serum exosomes derived from patients with myocardial ischemia regulate angiogenesis through the miR-939-mediated nitric oxide signaling pathway. Theranostics. (2018) 8:2079-93. doi: 10.7150/thno. 21895

44. Liu S, Chen J, Shi J, Zhou W, Wang L, Fang W, et al. M1-like macrophagederived exosomes suppress angiogenesis and exacerbate cardiac dysfunction in a myocardial infarction microenvironment. Basic Res Cardiol. (2020) 115:22. doi: $10.1007 / \mathrm{s} 00395-020-0781-7$

45. Ibrahim AG, Cheng K, Marbán E. Exosomes as critical agents of cardiac regeneration triggered by cell therapy. Stem Cell Rep. (2014) 2:60619. doi: $10.1016 /$ j.stemcr.2014.04.006 
46. Ribeiro-Rodrigues TM, Laundos TL, Pereira-Carvalho R, Batista-Almeida D, Pereira R, Coelho-Santos V, et al. Exosomes secreted by cardiomyocytes subjected to ischaemia promote cardiac angiogenesis. Cardiovasc Res. (2017) 113:1338-50. doi: $10.1093 / \mathrm{cvr} / \mathrm{cvx} 118$

47. Pfeifer P, Werner N, Jansen F. Role and function of microRNAs in extracellular vesicles in cardiovascular biology. Biomed Res Int. (2015) 2015:161393. doi: 10.1155/2015/161393

48. Goren Y, Kushnir M, Zafrir B, Tabak S, Lewis BS, Amir O. Serum levels of microRNAs in patients with heart failure. Eur J Heart Fail. (2012) 14:14754. doi: 10.1093/eurjhf/hfr155

49. Schneider SIDR, Silvello D, Martinelli NC, Garbin A, Biolo A, Clausell N, et al. Plasma levels of microRNA-21,-126 and-423-5p alter during clinical improvement and are associated with the prognosis of acute heart failure. $\mathrm{Mol}$ Med Rep. (2018) 17:4736-46. doi: 10.3892/mmr.2018.8428

50. Endo K, Naito Y, Ji X, Nakanishi M, Noguchi T, Goto Y, et al. MicroRNA 210 as a biomarker for congestive heart failure. Biol Pharm Bull. (2013) 36:48-54. doi: 10.1248/bpb.b12-00578

51. Ellis KL, Cameron VA, Troughton RW, Frampton CM, Ellmers LJ, Richards AM. Circulating microRNAs as candidate markers to distinguish heart failure in breathless patients. Eur J Heart Fail. (2013) 15:113847. doi: 10.1093/eurjhf/hft078

52. Corsten MF, Dennert R, Jochems S, Kuznetsova T, Devaux Y, Hofstra L, et al. Circulating microRNA-208b and microRNA-499 reflect myocardial damage in cardiovascular disease. Circ Cardiovasc Genet. (2010) 3:499506. doi: 10.1161/CIRCGENETICS.110.957415

53. Ovchinnikova ES, Schmitter D, Vegter EL, Ter Maaten JM, Valente MAE, Liu LCY, et al. Signature of circulating microRNAs in patients with acute heart failure. Eur J Heart Fail. (2016) 18:414-23. doi: 10.1002/ejhf.332

54. Stefanie K, Sebastian E, Jürgen P, Till N, Michael A, Frey UH. Circulating miR-192 is a prognostic marker in patients with ischemic cardiomyopathy. Fut Cardiol. (2018) 14:283-9. doi: 10.2217/fca-2017-0108

55. Lu W, Jiao L, Bin X, Yu-Lan L, Zhou L. Reduced exosome miR-425 and miR744 in the plasma represents the progression of fibrosis and heart failure. Kaohsiung J Med Sci. (2018) 34:626-33. doi: 10.1016/j.kjms.2018.05.008

56. Beg F, Wang R, Saeed Z, Devaraj S, Masoor K, Nakshatri H. Inflammationassociated microRNA changes in circulating exosomes of heart failure patients. BMC Res Notes. (2017) 10:751. doi: 10.1186/s13104-017-3090-y

57. Turchinovich A, Weiz L, Langheinz A, Burwinkel B. Characterization of extracellular circulating microRNA. Nucleic Acids Res. (2011) 39:722333. doi: $10.1093 /$ nar/gkr254

58. Wu T, Chen Y, Du Y, Tao J, Li W, Zhou Z, et al. Circulating exosomal miR$92 \mathrm{~b}-5 \mathrm{p}$ is a promising diagnostic biomarker of heart failure with reduced ejection fraction patients hospitalized for acute heart failure. J Thorac Dis. (2018) 10:6211-20. doi: 10.21037/jtd.2018.10.52

59. Sen M, Yasuhiko S, Shinichiro S, Daisaku N, Masaya U, Masahiko H, et al. Circulating p53-responsive microRNAs are predictive indicators of heart failure after acute myocardial infarction. Circ Res. (2013) 113:3226. doi: 10.1161/CIRCRESAHA.113.301209

60. Yang VK, Loughran KA, Meola DM, Juhr CM, Thane KE, Davis AM, et al. Circulating exosome microRNA associated with heart failure secondary to myxomatous mitral valve disease in a naturally occurring canine model. $J$ Extracell Vesicles. (2017) 6:1350088. doi: 10.1080/20013078.2017.1350088

61. Jansen F, Yang X, Proebsting S, Hoelscher M, Przybilla D, Baumann K, et al. MicroRNA expression in circulating microvesicles predicts cardiovascular events in patients with coronary artery disease. J Am Heart Assoc. (2014) 3:e1249. doi: 10.1161/JAHA.114.001249

62. Yuan Y, Du W, Liu J, Ma W, Zhang L, Du Z, et al. Stem cell-derived exosome in cardiovascular diseases: macro roles of micro particles. Front Pharmacol. (2018) 9:547. doi: 10.3389/fphar.2018.00547

63. Yang PC. Induced pluripotent stem cell (iPSC)-derived exosomes for precision medicine in heart failure. Circ Res. (2018) 122:6613. doi: 10.1161/CIRCRESAHA.118.312657

64. Wang X, Gu H, Qin D, Yang L, Huang W, Essandoh K, et al. Exosomal miR-223 contributes to mesenchymal stem cell-elicited cardioprotection in polymicrobial sepsis. Sci Rep. (2015) 5:13721. doi: 10.1038/srep 13721

65. Gray WD, French KM, Ghosh-Choudhary S, Maxwell JT, Brown ME, Platt $\mathrm{MO}$, et al. Identification of therapeutic covariant microRNA clusters in hypoxia-treated cardiac progenitor cell exosomes using systems biology. Circ Res. (2015) 116:255-63. doi: 10.1161/CIRCRESAHA.116.3 04360

66. Feng Y, Huang W, Wani M, Yu X, Ashraf M. Ischemic preconditioning potentiates the protective effect of stem cells through secretion of exosomes by targeting Mecp2 via miR-22. PLoS ONE. (2014) 9:e88685. doi: 10.1371/journal.pone.0088685

67. Mayourian J, Ceholski DK, Gorski PA, Mathiyalagan P, Murphy JF, Salazar SI, et al. Exosomal microRNA-21-5p mediates mesenchymal stem cell paracrine effects on human cardiac tissue contractility. Circ Res. (2018) 122:93344. doi: 10.1161/CIRCRESAHA.118.312420

68. Khan M, Nickoloff E, Abramova T, Johnson J, Verma SK, Krishnamurthy $\mathrm{P}$, et al. Embryonic stem cell-derived exosomes promote endogenous repair mechanisms and enhance cardiac function following myocardial infarction. Circ Res. (2015) 117:52-64. doi: 10.1161/CIRCRESAHA.117. 305990

69. Tang Y, Huang Y, Zheng L, Qin S, Xu X, An T, et al. Comparison of isolation methods of exosomes and exosomal RNA from cell culture medium and serum. Int J Mol Med. (2017) 40:834-44. doi: 10.3892/ijmm.201 7.3080

Conflict of Interest: The authors declare that the research was conducted in the absence of any commercial or financial relationships that could be construed as a potential conflict of interest.

Copyright (C) 2020 Xue, Tan, Wu, Dong, Xie, Huang, He, Dong and Liu. This is an open-access article distributed under the terms of the Creative Commons Attribution License (CC BY). The use, distribution or reproduction in other forums is permitted, provided the original author(s) and the copyright owner(s) are credited and that the original publication in this journal is cited, in accordance with accepted academic practice. No use, distribution or reproduction is permitted which does not comply with these terms. 